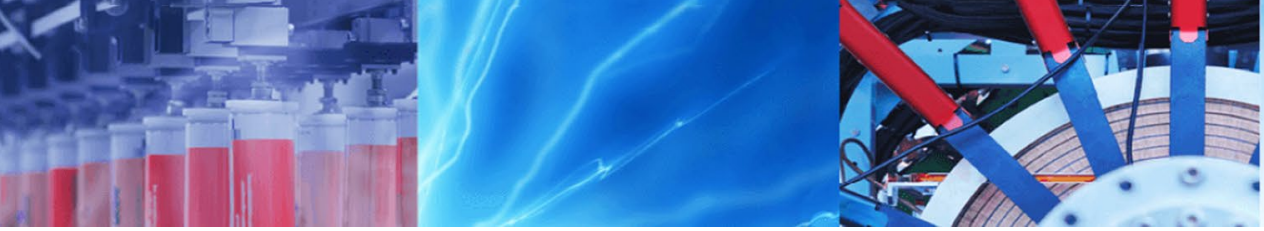

Research Article

\title{
Trapped flexural waves supported by a pair of identical cylinders in a two-layer fluid
}

\author{
Sunanda Saha ${ }^{1} \cdot$ Swaroop Nandan Bora ${ }^{2}$ (D)
}

Received: 7 April 2020 / Accepted: 20 July 2020 / Published online: 30 July 2020

(c) Springer Nature Switzerland AG 2020

\begin{abstract}
Existence of trapped waves in ocean shows the presence of discrete wave frequencies in the continuous spectrum. In the present work, we compute those trapped mode frequencies due to a pair of identical horizontal circular cylinders submerged in one of the layers of a two-layer fluid with a thin ice-cover at the upper layer and an infinite depth for the lower layer. Theory is developed for multiple cylinders but numerical computation is carried out only for a pair of cylinders. Due to a thin ice-cover replacing the free surface, a fifth-order boundary condition is to be considered in the upper layer that makes the problem complex and challenging but more practical. Considering linear water wave theory, the boundary value problem is developed through modified Helmholtz equation and associated conditions. Subsequently, applying multipole expansion method, an infinite system of homogeneous linear equations with complex coefficients is obtained and solved. By fixing the geometrical parameters and density ratio, the trapped mode frequencies are computed numerically by tracing the zeros of the determinant generated from the truncated system of the above mentioned equations. In the first instance, the cylinders are placed in the lower layer and the variation of trapped modes is examined by varying different parameters such as upper layer depth, submergence depth, ice-cover thickness etc. In the second instance, the same conditions and configurations are considered in the upper layer and existence of trapped modes is looked into in a similar manner. For the considered parameter values, the number of trapped modes enclosed in the continuous spectrum decreases corresponding to an increase in the flexural rigidity of the ice-cover. The trapped mode frequency decreases when either the the upper layer depth or the submergence depth increases. Further, corresponding to a small change in the separation parameter, embedded trapped modes are observed to cease to exist for the free surface and also for a very thin ice-cover. Our findings are supported by graphs depicting various modes. Further, comparison of present result with an established result shows excellent agreement.
\end{abstract}

Keywords Trapped mode $\cdot$ Two-layer fluid $\cdot$ Multipoles $\cdot$ Multiple cylinders

\section{Introduction}

Over the last few decades, an extensive research has taken place with an effort to understand the constraints for twodimensional linear water wave problems that give rise to a unique solution for a prescribed homogeneous boundary condition. Proving uniqueness is similar to establishing that there exist only trivial solutions for homogeneous boundary conditions. Partial results have been obtained by many researchers that establish the uniqueness of solutions for certain configurations, e.g., Simon and Ursell [31], Kuznetsov et al. [13]. Mclver [22] gave an example of non-uniqueness and showed that general uniqueness proof was unobtainable. She considered a pair of sources located on the free surface in a symmetric manner so that the sources were separated by half a wavelength to ensure

Swaroop Nandan Bora, swaroop@iitg.ac.in | 'Department of Mathematics, SAS, Vellore Institute of Technology, Vellore, India. ${ }^{2}$ Department of Mathematics, Indian Institute of Technology Guwahati, Guwahati 781039, India. 
that the potential did not radiate waves to infinity. Then, a difference velocity potential was constructed by using an inverse method that satisfied a specific linear water wave problem. This showed the existence of a non-trivial solution, whose scalar multiple would also be a solution, since the considered problem was linear. This non-trivial solution is known as 'trapped mode' as the energy gets trapped in the vicinity of the structures or bodies. The investigations carried out by Kuznetsov et al. [13] and Motygin [23] can be considered as extensions to this two-dimensional result. Numerical computation was carried out by Mclver and Evans [21] and Porter and Evans [28] to examine the presence of trapped modes for submerged horizontal cylinders. Most of these results, along with a number of generalizations and various other references, can be found in the book by Kuznetsov et al. [14] and article by Linton and Mclver [19]. Harter et al. [4,5] investigated the effect of surface tension on trapped modes and they showed that avoiding surface tension was not always appropriate since it was instrumental in changing the topological nature of the streamline pattern.

Linton and Evans [16] considered a numerical method to determine trapped modes for a bottom-mounted vertical cylinder of an arbitrary shape in finite water depth. Using Green's function method, the trapped modes were evaluated by using homogeneous system of Fredholm integral equations. Evans et al. [7] considered a long infinite vertical cylinder symmetric about the channel centreplane and established the existence of trapped modes for the anti-symmetric motion. The work of Evans et al. [7] involving a single cylinder was extended by Davies and Parnovski [3] for two identical cylinders-each being the reflection of the other in the centre-plane. They derived the existence and non-existence of trapped modes for specific shape, size and position of the structure(s). Porter [29] was instrumental in establishing a strong numerical evidence in favor of the existence of two-dimensional trapped waves by considering a pair of symmetric horizontal submerged cylinders. Recently, Tabssum et al. [35] considered gravity wave interaction with a porous breakwater in a two-layer ocean of varying depth and discussed both scattering and trapping of waves. This study has enhanced the understanding of scattering as well as trapping of waves by a porous breakwater under wave-wave interaction in a two-layer ocean of continental shelf.

Study of ice-cover dynamics has gained its popularity because of increase in various activities in Arctic and Antarctic regions. To analyze the effect of wave propagation through the nearly continuous ice-cover of those two regions, ice-cover is approximated as a thin elastic sheet partially immersed in water. As a consequence, the associated upper surface boundary condition for the problem does not take the form of the standard Sturm-Liouville type that occurs in case of plane gravity waves. Fox and Squire [9] considered an oblique wave at the ice-cover edge and derived a linearized model for the whole scattering process. Chung and Fox [2] considered the interaction of the propagating waves with a semi-infinite ice sheet and determined the reflection coefficient of incident waves. Further, Evans and Porter [8], by using Green's function approach, investigated the scattering of oblique incident waves by a narrow crack of an ice-sheet floating on the upper surface of the ocean.

Layered fluid models can be considered as a one-step advanced approximation of realistic stratified fluids. These models can be used to investigate the existence of trapped waves near submerged bodies. These models will give rise to interfaces between the layers of the fluid which can be considered as free surface for the internal waves. In order to accomplish this, linear water wave theory can be appropriately employed layer-wise under the assumptions of immiscible and gravitationally stable layers with constant densities. As an example, modeling of large-scale atmospheric and oceanic flows with shallow-water dynamics and estuarine dynamics can be accomplished with the consideration of a simple two-layer model.

Kuznetsov [12] was the first one to prove the existence of trapped modes in a two-layer fluid. He considered a setup consisting of a submerged cylinder located in infinite depth lower layer with the assumption of small density difference between the layers. Using perturbation technique, the existence of these modes on both the free surface and the interface was studied. Later, the trapped mode frequencies were computed by Linton and Cadby [18] for a horizontal circular cylinder submerged in one of the layers. They also computed discrete trapped wave frequencies in the continuous spectrum for two identical circular cylinders submerged in the lower layer. Nazarov and Videman [26] examined the existence of trapped waves for a submerged body in a two-layer fluid when it neither intersected the free surface nor the interface and they established the general sufficient condition for trapped water waves. Xu and Lu [37] discussed the hydroelastic interaction between an incident gravity wave and a thin elastic plate floating in a two-layer fluid of constant depth. They discussed the effect of density ratio of the fluids and the position of the interface on reflection and transmission of the wave. Nazarov et al. [27] investigated the trapping of oblique water waves by horizontal cylinders in a two-layer liquid for two cases depending on a small parameter. They provided asymptotic formulas for the surface and interfacial trapped mode frequencies. Saha and Bora [33] studied the existence of trapped waves for a horizontal circular cylinder submerged in one of the layers of a two-layer fluid of finite depth with layer-wise constant densities when the fluid was covered by a rigid lid. Romero Rodriguez and 
Zhevandrov [30] produced exact solutions for oblique water waves trapped by a submerged horizontal cylinder of small but arbitrary cross-section in a two-layer fluid. The solutions were developed in terms of convergent series in powers of the small parameter which signified the thinness of the cylinder.

It has been established that two progressive waves can be obtained at the upper surface and the interface for a two-layer fluid with a thin ice-cover. The following works provide ample insight in this direction: (1) Bhattacharjee and Sahoo [32] considered flexural gravity wave problems in a two-layer fluid and obtained Fourier type expansion formulas along with the related orthogonal mode-coupling relations; (2) Mohapatra and Bora [24] investigated the propagation of oblique waves over a small bottom undulation in an ice-covered two-layer fluid. The reflection and transmission coefficients were determined by solving the modified Helmholtz equation; (3) Mohapatra and Bora [25] took up the scattering problem for a submerged sphere placed in one of the layers of a two-layer fluid in finite depth covered by a thin ice-cover and computed the exciting forces in both horizontal and vertical directions. (4) Saha and Bora [34], by following assumptions of linear water wave theory, established the existence of trapped modes supported by a submerged horizontal circular cylinder in a two-layer fluid of finite depth bounded above by a thin ice-cover and below by an impermeable horizontal bottom. They found that above a certain cut-off frequency and far from interface and ice-cover, it was possible to have a unique solution to the radiation problem for the cylinder placed in either of the layers.

In recent times, Hughes et al. [10] have provided a lot of information on the role of coastal trapped waves in mediating the influence of open ocean on the coast. This article lucidly describes many aspects of ocean, waves and continental shelf, etc.

Over the years, the study of flexural gravity wave propagation in a two-layer fluid has become very important as illustrated by the above works and therefore, this topic has gained huge impetus for research to be carried out.

However, as far as the knowledge of the current authors is concerned, the study on trapped waves for a pair of identical circular cylinders placed in a two-layer fluid covered by a thin ice-sheet is not available in the literature till date. Though the current authors investigated various aspects of existence of flexural trapped waves earlier in [34], it was restricted to a single cylinder placed in one of the layers of a two-layer fluid. The objective of the present work is to locate the distance between these two identical cylinders for which trapped wave exists. The variation of this distance is observed by varying the ice parameters values, the depth of the upper layer and the submergence depth when the pair of cylinders is placed in the lower layer. The case of the cylinders being placed in the upper layer is also considered and the distances are located for which trapped wave exists. This article is arranged as follows: In Sect. 2, the problem is formulated and the trapped modes are determined which may occur for the arrangement of any number of horizontal circular cylinders placed on a horizontal plane with their axes parallel to each other. In Sect. 3, the multipole expansion method adopted by Kassem [11] is used so that the singular solutions of the modified Helmholtz equation satisfies all the prescribed boundary conditions. The total velocity potential is written as a linear combination of all relevant multipoles. Using body boundary conditions on the surfaces of the cylinders, an infinite system of homogeneous linear equations is derived. After truncating the system, the non-trivial solutions indicate the trapped modes. Section 4 presents the results and discussions for two identical cylinders placed in either of the layers in addition to validation. The significance of the present work and its contribution is briefly discussed in Sect. 5. At the end, all results are summarized in Sect 6.

\section{Mathematical formulation}

A two-layer inviscid, incompressible and immiscible fluid with irrotational motion of relatively small amplitude is considered in which the upper layer of depth $d$ is covered by a thin uniform ice-sheet and the lower layer is of infinite depth. The surface tension effect is neglected at the interface of the layers and each fluid layer is of infinite horizontal extent in both directions of the $x y$-plane while finite depth is considered along the $z$-direction with vertical upward positive orientation by taking $z=d>0$ as the mean position of the thin ice-sheet and $z=0$ as the mean position of the interface (Fig. 1). With these considerations, velocity potentials in the lower and the upper layers for oblique waves can be written, respectively, in the following form:

$$
\begin{aligned}
& \Phi^{\prime}(x, y, z, t)=\operatorname{Re}\left[\phi^{\prime}(x, z) e^{i l y} e^{-i \omega t}\right] \quad \text { and } \\
& \Phi^{\prime \prime}(x, y, z, t)=\operatorname{Re}\left[\phi^{\prime \prime}(x, z) e^{i l y} e^{-i \omega t}\right],
\end{aligned}
$$

with $\phi^{\prime}(x, z)$ denoting complex-valued potential for the upper layer fluid $(-\infty<x<\infty, 0<z<d)$ of density $\rho$; $\phi^{\prime \prime}(x, z)$ the complex-valued potential for the lower layer fluid $(-\infty<x<\infty,-\infty<z<0)$ of higher density $\rho_{\| \prime}$; $I$ the $y$-component of the wave number; $\omega$ the angular frequency of the incoming wave; Re the real part of the bracketed quantity and $i=\sqrt{-1}$.

Governing equations for the velocity potentials $\phi^{\prime}$ and $\phi^{\prime \prime}$ are modified Helmholtz equation as follows: 


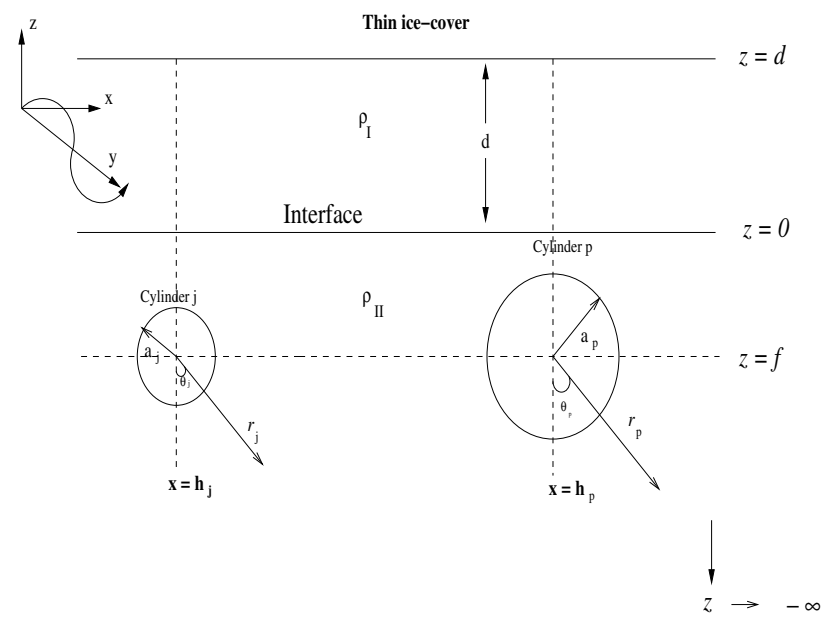

Fig. 1 Schematic diagram of two cylinders in the lower layer with the upper layer covered by an ice-cover

$\left(\nabla_{x, z}^{2}-l^{2}\right) \phi^{\prime}=0$ for the upper fluid layer,

$\left(\nabla_{x, z}^{2}-l^{2}\right) \phi^{\prime \prime}=0 \quad$ for the lower fluid layer.

Let $\rho$ be the ratio $\rho_{1} / \rho_{\| \prime}(<1)$ of the densities of the two fluids. Subsequently, the boundary conditions at the interface and the ice-cover can be written as

$\frac{\partial \phi^{\prime}}{\partial z}=\frac{\partial \phi^{\prime \prime}}{\partial z} \quad$ on $z=0$,

$\rho\left(\frac{\partial \phi^{\prime}}{\partial z}-K \phi^{\prime}\right)=\frac{\partial \phi^{\prime \prime}}{\partial z}-K \phi^{\prime \prime} \quad$ on $z=0$,

$\left[D\left(\frac{\partial^{2}}{\partial x^{2}}-l^{2}\right)^{2}+1-\epsilon K\right] \frac{\partial \phi^{\prime}}{\partial z}-K \phi^{\prime}=0 \quad$ on $z=d$,

where $K=\omega^{2} / g$ with $g$ as the gravitational constant; $D=L /(\rho, g)$ with $L$ being the flexural rigidity of the elastic ice-cover given by $L=E h_{0}^{2} /\left[12\left(1-\gamma^{2}\right)\right] ; E$ and $\gamma$, respectively, denote Young's modulus and Poisson's ratio for ice; $h_{0}$ the very small thickness of the ice-cover; $\epsilon=\left(\rho_{0} / \rho_{1}\right) h_{0}$ with $\rho_{0}$ denoting the density of the ice. As usual, the boundary conditions (2.4) and (2.5), respectively, imply the continuity of normal velocity and pressure at the interface. For detailed derivation of the ice-cover boundary condition (2.6), readers are referred to the Appendix of [34].

Because of consideration of infinite depth lower layer, the following limiting values must be valid:

$\phi^{\prime \prime},\left|\nabla \phi^{\prime \prime}\right| \rightarrow 0 \quad$ as $z \rightarrow-\infty$.
Under this set-up, incident waves, for an arbitrary multiplicative constant, can be written as

$$
\begin{aligned}
& \phi^{\prime}=\exp \left( \pm i x \sqrt{u^{2}-l^{2}}\right)\left(F_{+}(u) e^{u(z-d)}+F_{-}(u) e^{-u(z-d)}\right), \\
& \phi^{\prime \prime}=\exp \left( \pm i x \sqrt{u^{2}-I^{2}}\right) e^{u z}\left(F_{+}(u) e^{-u d}-F_{-}(u) e^{u d}\right),
\end{aligned}
$$

where

$F_{ \pm}(u)=\left(D u^{4}+1-\epsilon K\right) u \pm K$,

with $u$ satisfying the dispersion relation

$(u-K \sigma) F_{-}(u)-(u-K) F_{+}(u) e^{-2 u d}=0$,

for $\sigma=(1+\rho) /(1-\rho)$.

It may be noted that Lu and Sun [20] derived an explicit analytical solution of dispersion relations of the type (2.9).

By fixing the geometrical parameters and the density ratio, it is known, based on the work of Bhattacharjee and Sahoo [32], that exactly two positive real roots $u_{1}$ and $u_{2}$ $\left(u_{1}<u_{2}\right.$, say) can be obtained for a specific value of $K$. This equation gets reduced to the dispersion relation derivable for a two-layer fluid of infinite depth bounded above by a free surface, i.e., for the specific case of $D=\epsilon=0$.

\section{Finding solutions by method of multipoles}

$N$ fixed horizontal circular cylinders of infinite length are placed on the plane $z=f$ with their generators running parallel to the $y$-axis. For the case $f>0$, the cylinders are located entirely in the upper fluid while for $f<0$, the cylinders are entirely located in the lower fluid. The radius of the $j$-th cylinder is taken as $a_{j}$ and its center is positioned at $(x, z)=\left(h_{j}, f\right), j=1,2, \ldots, N$. For convenience, local polar coordinates $\left(r_{j}, \theta_{j}\right)$ associated with cylinder $j$ are defined by (Fig. 1)

$x_{j}=h_{j}+r_{j} \sin \theta_{j} \quad$ and $\quad z_{j}=f-r_{j} \cos \theta_{j}$.

\subsection{Cylinders submerged in lower layer only}

\subsubsection{Arbitrary single cylinder}

Here we consider an arbitrary cylinder of radius $a_{j}$ for $j=1,2, \ldots, N$. The expressions for the symmetric and antisymmetric multipoles, $\phi_{n j}^{s}(n \geq 0)$ and $\phi_{n j}^{a}(n \geq 1)$, respectively, are given by, as detailed in [17],

$$
\begin{aligned}
\phi_{n j}^{l s}\left(x_{j}, z_{j}\right)= & (-1)^{n} \int_{0}^{\infty} \cosh n u \cos \left(l x_{j} \sinh u\right)\left[A_{L}(v) e^{v z_{j}}\right. \\
& \left.+B_{L}(v) e^{-v z_{j}}\right] d u
\end{aligned}
$$


$\phi_{n j}^{1 / s}\left(x_{j}, z_{j}\right)=K_{n}\left(I r_{j}\right) \cos n \theta_{j}+(-1)^{n} \int_{0}^{\infty} \cosh n u \cos \left(I x_{j} \sinh u\right) e^{v z_{j}} C_{L}(v) d u$,

$\phi_{n j}^{\prime a}\left(x_{j}, z_{j}\right)=(-1)^{n+1} \int_{0}^{\infty} \sinh n u \sin \left(l x_{j} \sinh u\right)\left[A_{L}(v) e^{v z_{j}}+B_{L}(v) e^{-v z_{j}}\right] d u$,

$\phi_{n j}^{\| l a}\left(x_{j}, z_{j}\right)=K_{n}\left(I r_{j}\right) \sin n \theta_{j}+(-1)^{n+1} \int_{0}^{\infty} \sinh n u \sin \left(I x_{j} \sinh u\right) e^{v z_{j}} C_{L}(v) d u$,

where $v=I \cosh u ; K_{n}($.$) is the n$-th order modified Bessel function of second kind and

$A_{L}(v)=\frac{F_{+}(v)}{F_{-}(v)} B_{L}(v) e^{-2 v d}, \quad B_{L}(v)=\frac{K(1+\sigma) F_{-}(v)}{G(v)} e^{v f}$,

$C_{L}(v)=\frac{B_{L}(v)}{K(1+\sigma) F_{-}(v)}\left[(v+K \sigma) F_{+}(v) e^{-2 v d}-(v+K) F_{-}(v)\right]$,

with

$G(v)=(v-K \sigma) F_{-}(v)-(v-K) F_{+}(v) e^{-2 v d}$.

The functions $\phi_{n j}^{s}$ and $\phi_{n j}^{a}$ are the singular solutions to the modified Helmholtz equation satisfying all the boundary conditions except for the body boundary condition. Since $u_{1}$ and $u_{2}$ are roots of the dispersion relation (2.9), the multipole potentials have two simple poles $u=v_{1}$ and $u=v_{2}$, where

$l \cosh v_{1}=u_{1} \quad$ and $\quad l \cosh v_{2}=u_{2}$.

Subsequently, all the integrals appearing in Eqs. (3.2)-(3.5) are complex-valued.

Now we expand the multipoles in polar coordinates by taking $X=-I r_{j}$ and $T=\exp \left[i\left(\theta_{j}+i u\right)\right]$ in Eq. (A.4) of "Appendix $A$ " and then by considering the real and imaginary parts, those expressions can be put into Eqs. (3.3) and (3.5) to obtain the following:

$\phi_{n j}^{1 / s}\left(r_{j}, \theta_{j}\right)=K_{n}\left(I r_{j}\right) \cos n \theta_{j}+\sum_{m=0}^{\infty} A_{m n}^{s} I_{m}\left(I r_{j}\right) \cos m \theta_{j}$

$\phi_{n j}^{I I a}\left(r_{j}, \theta_{j}\right)=K_{n}\left(I r_{j}\right) \sin n \theta_{j}+\sum_{m=0}^{\infty} A_{m n}^{a} I_{m}\left(I r_{j}\right) \sin m \theta_{j}$,

where $I_{n}($.$) is the n$th order modified Bessel function of first kind and
$A_{m n}^{s}=\varepsilon_{m}(-1)^{m+n} \int_{0}^{\infty} \cosh m u \cosh n u e^{v f} C_{L}(v) d u$,

$A_{m n}^{a}=2(-1)^{m+n} \int_{0}^{\infty} \sinh m u \sinh n u e^{v f} C_{L}(v) d u$,

with $\varepsilon_{0}=1, \varepsilon_{n}=2, n \geq 1$. From computational point of view, the contour integral appearing in the above coefficients can be appropriately written as the sum of the principal value integral and the residual contribution corresponding to the simple poles $v_{1}$ and $v_{2}$. The principal value integrals can be evaluated by using the method described in Linton and Evans [15].

\subsubsection{Multiple cylinders}

We now construct a possible trapped mode potential as the sum over all the multipoles and also for all the cylinders:

$\phi^{\prime \prime}(r, \theta)=\sum_{j=1}^{N} \sum_{n=0}^{\infty}\left(A_{n}^{j} \phi_{n j}^{\prime l s}\left(r_{j}, \theta_{j}\right)+B_{n}^{j} \phi_{n j}^{\prime \prime a}\left(r_{j}, \theta_{j}\right)\right)$,

for some constants $A^{j}$ and $B^{j}$ by searching for the possible non-trivial solutions which satisfy the body boundary condition on the cylinders given by

$\frac{\partial \phi^{\prime \prime}}{\partial r_{j}}=0 \quad$ on $\quad r_{j}=a_{j}, j=1, \ldots, N$.

For imposing this important condition, it is required to shift from the individual coordinates of each cylinder $j$ to those of a fixed cylinder $p$, say, where $p \neq j$ (Fig. 1). By doing so, the following important results are obtained ("Appendix A"): 


$$
\begin{aligned}
& K_{n}\left(I r_{j}\right) \cos n \theta_{j}=\sum_{m=0}^{\infty}\left(C_{n m}^{j p} \cos m \theta_{p}+D_{n m}^{j p} \sin m \theta_{p}\right) I_{m}\left(I r_{p}\right), \\
& K_{n}\left(I r_{j}\right) \sin n \theta_{j}=\sum_{m=0}^{\infty}\left(A_{n m}^{j p} \cos m \theta_{p}+B_{n m}^{j p} \sin m \theta_{p}\right) I_{m}\left(I r_{p}\right),
\end{aligned}
$$

and

$$
\begin{aligned}
& (-1)^{n} \int_{0}^{\infty} \cosh n u \cos \left(I x_{j} \sinh u\right) e^{v z_{j}} C_{L}(v) d u \\
& =\sum_{m=0}^{\infty}\left(\alpha_{n m}^{j p} \cos m \theta_{p}+\beta_{n m}^{j p} \sin m \theta_{p}\right) I_{m}\left(I r_{p}\right), \\
& (-1)^{n+1} \int_{0}^{\infty} \sinh n u \sin \left(I x_{j} \sinh u\right) e^{v z_{j}} C_{L}(v) d u \\
& =\sum_{m=0}^{\infty}\left(a_{n m}^{i p} \cos m \theta_{p}+b_{n m}^{j p} \sin m \theta_{p}\right) I_{m}\left(I r_{p}\right) .
\end{aligned}
$$

It follows from (3.3), (3.5) and ()-(3.13) that for $j \neq p$,

$\phi_{n j}^{2 s}\left(r_{j}, \theta_{j}\right)=\sum_{m=0}^{\infty}\left[\left(C_{n m}^{j p}+\alpha_{n m}^{j p}\right) \cos m \theta_{p}+\left(D_{n m}^{j p}+\beta_{n m}^{j p}\right) \sin m \theta_{p}\right] I_{m}\left(I r_{p}\right)$,

$\phi_{n j}^{2 a}\left(r_{j}, \theta_{j}\right)=\sum_{m=0}^{\infty}\left[\left(A_{n m}^{j p}+a_{n m}^{j p}\right) \cos m \theta_{p}+\left(B_{n m}^{j p}+b_{n m}^{j p}\right) \sin m \theta_{p}\right] I_{m}\left(I r_{p}\right)$.

For the case $j=p$, the above multipoles take the form given by (3.8) and (3.9). We are now in a position to write

$$
\phi^{\prime \prime}(r, \theta)=\sum_{n=0}^{\infty}\left(A_{n}^{p} \phi_{n p}^{1 / 5}+B_{n}^{p} \phi_{n p}^{\prime \prime a}\right)+\sum_{\substack{j=1 \\ j \neq p}}^{N} \sum_{n=1}^{\infty}\left(A_{n}^{j} \phi_{n j}^{1 / 5}+B_{n}^{j} \phi_{n j}^{\prime \prime a}\right) .
$$

Subsequently, by imposing (3.11) and using the orthogonality property of sine and cosine functions, we obtain frequencies for which zeros of the truncated determinant exist. Though the theoretical development derived above is valid for any arbitrary number of cylinders, but for examining the variation of trapped modes, it is considered convenient to consider a pair of identical cylinders in the next section. The result then can subsequently be extended to any number of cylinders. For the case of cylinders placed on $z=f, f>0$, i.e., when the cylinders are submerged in the upper layer, we can proceed in a similar way and again obtain the trapped mode frequency by solving the truncated system of infinite homogeneous linear equations.

\section{Numerical results and discussion for two identical cylinders submerged in either of the layers}

For the case of two identical cylinders $\left(a_{1}=a_{2}=a\right)$ placed at $h_{1}=-h_{2}=\xi$, we can exploit the geometry and consider only the oscillations symmetric about $x=0$ so that the configuration can be considered equivalent to a horizontal cylinder placed next to a vertical wall. For the case of the upper layer covered by a free surface, Linton and Cadby [17] showed that the zeros of transmission for the oblique wave scattering problem occurred just below the cut-off frequency. Thereafter they considered the region $K<I<k$ and demonstrated the existence of embedded trapped modes [18]. In this region, propagating waves exist with wavenumber $k$ but not with wavenumber $K$. When a thin ice-cover plays the role in place of gravity waves on the upper surface, it was shown by Das and Mandal [6] that the maximum reflection occurred for frequencies just below the cutoff frequency of the incident wave of wavenumber $u_{2}$.

$$
\begin{aligned}
A_{m}^{p}+\frac{l_{m}^{\prime}\left(l a_{p}\right)}{K_{m}^{\prime}\left(l a_{p}\right)} \sum_{n=0}^{\infty}\left[A_{n}^{p} A_{m n}^{s}+\sum_{\substack{j=1 \\
j \neq p}}^{N}\left[A_{n}^{j}\left(C_{n m}^{j p}+\alpha_{n m}^{j p}\right)+B_{n}^{j}\left(A_{n m}^{j p}+a_{n m}^{j p}\right)\right]\right]=0, \\
B_{m}^{p}+\frac{l_{m}^{\prime}\left(I a_{p}\right)}{K_{m}^{\prime}\left(I a_{p}\right)} \sum_{n=0}^{\infty}\left[B_{n}^{p} A_{m n}^{a}+\sum_{\substack{j \\
j}}^{N}\left[A_{n}^{j}\left(D_{n m}^{j p}+\beta_{n m}^{j p}\right)+B_{n}^{j}\left(B_{n m}^{j p}+b_{n m}^{j p}\right)\right]\right]=0,
\end{aligned}
$$

where $p=1,2, \ldots, N ; m \geq 0$. Non-trivial solutions of this system allow us to determine the trapped mode frequencies due to the presence of $N$ cylinders arranged as described earlier. For obtaining non-trivial solutions of the unknown coefficients, it is required to determine the
Hence for seeking the embedded trapped modes, the region $u_{1}<I<u_{2}$ must be considered. 


\subsection{Cylinders submerged in the lower layer}

Since our interest lies in finding an even or a symmetric solution about $x=0$, therefore we consider

$\frac{\partial \phi^{\prime \prime}}{\partial x}=0 \quad$ on $\quad x=0$.

Condition (4.1) will be satisfied if $A_{n}^{1}=A_{n}^{2}$ and $B_{n}^{1}=-B_{n}^{2}$. Use of this reduces the original coupled system (3.16) simply to

$$
\begin{aligned}
& A_{m}^{1}+\frac{l_{m}^{\prime}(l a)}{K_{m}^{\prime}(l a)} \sum_{n=0}^{\infty}\left(P_{n m} A_{n}^{1}+Q_{n m} B_{n}^{1}\right)=0, \\
& B_{m}^{1}-\frac{l_{m}^{\prime}(l a)}{K_{m}^{\prime}(l a)} \sum_{n=0}^{\infty}\left(T_{n m} B_{n}^{1}+R_{n m} A_{n}^{1}\right)=0, m \geq 0,
\end{aligned}
$$

where

$$
\begin{aligned}
P_{n m} & =\frac{\varepsilon_{m}}{2}\left(K_{n-m}(2 \mid \xi)+(-1)^{m} K_{n+m}(2 / \xi)\right) \cos (n+m) \frac{\pi}{2} \\
& +\varepsilon_{m}(-1)^{n+m} \int_{0}^{\infty} \cosh n u \cosh m u e^{v f}(1+\cos (2 l \xi \sinh u)) C_{L}(v) d u, \\
Q_{n m} & =\frac{\varepsilon_{m}}{2}\left(K_{n-m}(2 \mid \xi)+(-1)^{m} K_{n+m}(2 / \xi)\right) \sin (n+m) \frac{\pi}{2} \\
& +\varepsilon_{m}(-1)^{n+m+1} \int_{0}^{\infty} \sinh n u \cosh m u e^{v f} \sin (2 / \xi \sinh u) C_{L}(v) d u, \\
R_{n m} & =-\frac{\varepsilon_{m}}{2}\left(K_{n-m}(2 / \xi)+(-1)^{m+1} K_{n+m}(2 / \xi)\right) \sin (n+m) \frac{\pi}{2} \\
& +\varepsilon_{m}(-1)^{n+m} \int_{0}^{\infty} \sinh m u \cosh n u e^{v f} \sin (2 / \xi \sinh u) C_{L}(v) d u, \\
T_{n m} & =\frac{\varepsilon_{m}}{2}\left(K_{n-m}(2 / \xi)+(-1)^{m+1} K_{n+m}(2 / \xi)\right) \cos (n+m) \frac{\pi}{2} \\
& +\varepsilon_{m}(-1)^{n+m} \int_{0}^{\infty} \sinh n u \sinh m u e^{v f}(\cos (2 / \xi \sinh u)-1) C_{L}(v) d u .
\end{aligned}
$$

determinant of matrix A vanishes. To determine such frequencies, we truncate the matrix $\mathbf{A}$ to a $2 M \times 2 M$ one and compute the determinant. Repetition of this process will yield the solution for symmetrical arrangements involving larger numbers of cylinders. In our following computation, we consider $M=8$.

\subsubsection{Numerical results}

An oblique wave of wavenumber $u_{2}$ propagating from the negative $x$-direction and making an angle $\alpha_{i n c}$ to the positive $x$-axis of the form $\exp [i b(x-\xi)]$, $b=\sqrt{u_{2}^{2}-l^{2}}=u_{2} \cos \alpha_{i n c}$ is considered to be incident on the cylinder centered at $(\xi, f)$ with the assumption that the second cylinder does not alter the interaction between the wave and the first cylinder. After fixing the geometrical parameters and density ratio, the evaluation of trapped
It is to be noted that $C_{L}(v)$ has only one singularity on the real axis at $v_{2}$ while it does not have any at $v_{1}$. Further, the path of integration is indented beneath this pole.

This system of equations can be written in matrix form as follows:

$$
\mathbf{A x}=\left(\begin{array}{ll}
\mathbf{P}+\mathbf{I} & \mathbf{Q} \\
\mathbf{R} & \mathbf{T}-\mathbf{I}
\end{array}\right)\left[\begin{array}{l}
\mathbf{a} \\
\mathbf{b}
\end{array}\right]=0
$$

where

$$
\begin{array}{rlrl}
\mathbf{P} & =\left[I_{m}^{\prime}(l a) P_{n m} / K_{m}^{\prime}(l a)\right], & \mathbf{Q}=\left[I_{m}^{\prime}(l a) Q_{n m} / K_{m}^{\prime}(l a)\right], \\
\mathbf{R}=\left[I_{m}^{\prime}(l a) R_{n m} / K_{m}^{\prime}(l a)\right], & \mathbf{T}=\left[I_{m}^{\prime}(l a) T_{n m} / K_{m}^{\prime}(l a)\right], \\
\mathbf{a}=\left[A_{m}^{\prime}\right], \quad \mathbf{b}=\left[B_{m}^{1}\right], & &
\end{array}
$$

for $m \geq 0$ and $n \geq 0$. In order to find non-trivial solutions, it is required to find the frequencies for which the mode frequencies entirely depends on two non-dimensional parameters $K a$ and $\xi / a$. Considering $\xi / a$, the parameter $K a$ is varied to locate the zeros of the real part of the truncated determinant. Subsequently, corresponding to those values of $K a$, the absolute values of the determinant are plotted. In all the figures, the fixed values considered are $\rho=0.50$ and $\alpha_{i n c}=0.34$. It is to be noted that the angle $\alpha_{i n c}$ is such that there are no propagating waves in the upper layer for all $\mathrm{Ka}$. Also, it is known that for a two-layer fluid consisting of fresh water and salt water, the ideal value of $\rho$ is around 0.97 . If this density ratio is considered, the same qualitative features are observed for our work but the effects of the interface do not come out very distinct. Therefore, we consider $\rho=0.5$ for our problem in order to have a better visual observation.

When both the cylinders are in the lower layer only, the $\xi / a$ values are considered up to 8.0 and then 


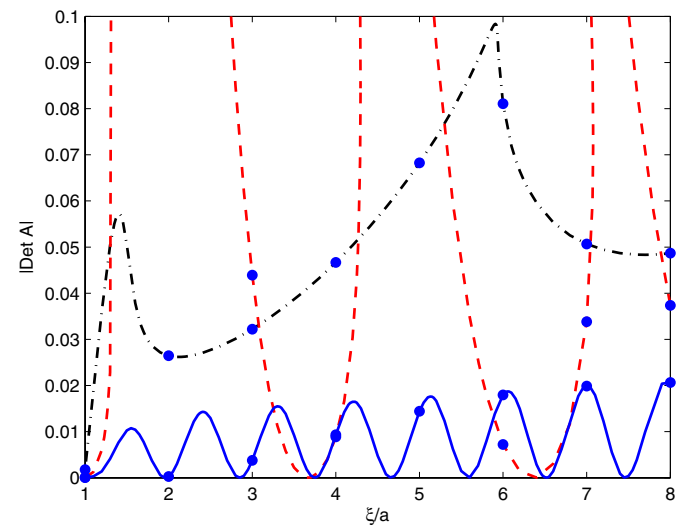

(a)

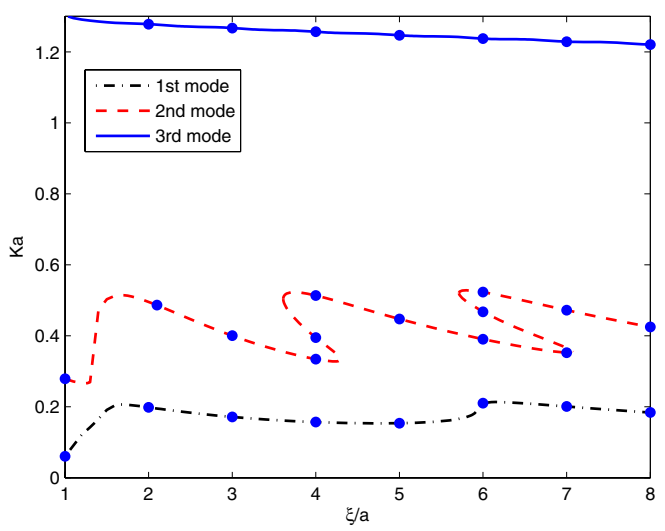

(b)
Fig. 2 a Values of $K a$ for which the real part of the determinant vanishes and $\mathbf{b}$ the absolute values of the determinant of the complex matrix for two cylinders of equal radius $a$ submerged in the

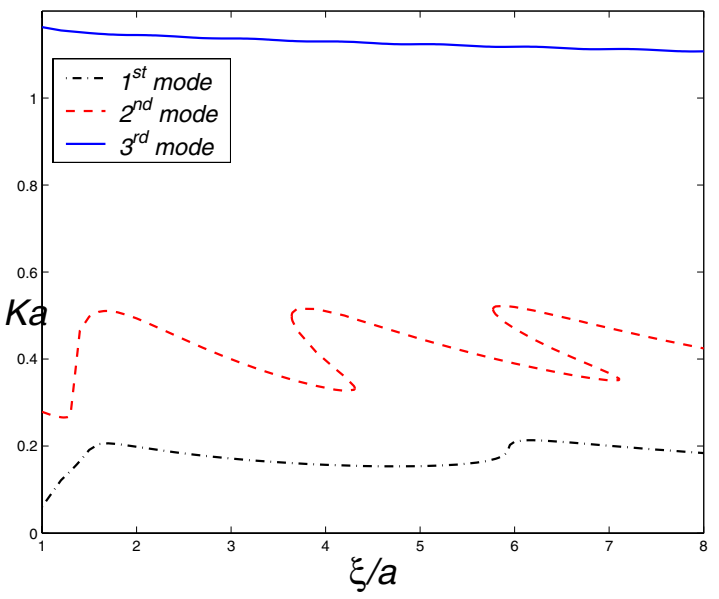

(a)

Fig. 3 a Values of $K a$ for which the real part of the determinant vanishes and $\mathbf{b}$ the absolute values of the determinant of the complex matrix for two cylinders of equal radius $a$ submerged in the lower

the variation of trapped frequencies is observed for $\xi / a \in[1,8]$. In Figs. 2,3 and 4 , the depth $d / a$ of the upper layer is taken as 2.0 and the submergence depth $f / a$ as - 1.1. In Fig. 2, $K a$ is plotted against $\xi / a$ with the values of the ice parameters as zero which actually depicts the free surface problem investigated earlier by Linton and Cadby [18] which is also shown along with the present result for comparison. The depiction in the figures clearly shows that the results of Linton and Cadby [18] and the present work converge to an absolute agreement. Since the work in [18] is considered to be a benchmark result, the proper validation of the present work with it lower layer; $d / a=2, f / a=-1.1, \rho=0.5, \alpha_{i n c}=0.34, D / a^{4}=0$ and $\epsilon / a=0$. Comparison of present work with Linton and Cadby [18]

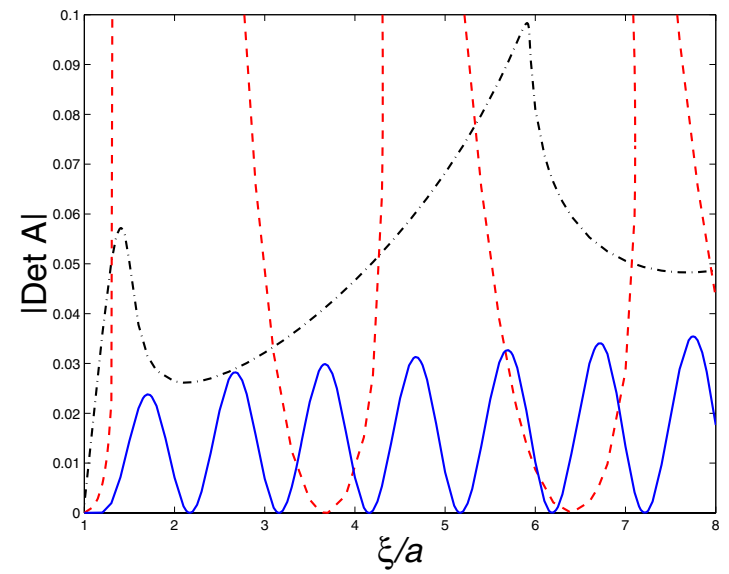

(b)

layer; $d / a=2, f / a=-1.1, \rho=0.5, \alpha_{i n c}=0.34, D / a^{4}=0.001$ and $\epsilon / a=0.001$

confirms that the work carried out is a correct one and hence it is reasonable to carry out subsequent investigations with conviction (in Fig. 2, the continuous curves represent the present work while the bullets represent the work of Linton and Cadby [18]).

The curves in part (a) of this figure as well in all the subsequent figures will be treated as modes.

In Figs. 3 and 4 , the flexural rigidity $D / a^{4}$ is considered, respectively, as 0.001 and 0.01 - a distinct change from Fig. 2 and this implies that a thin ice-cover has been taken into account in place of a free surface. It is observed from Figs. 3 and 4, upon this replacement, 


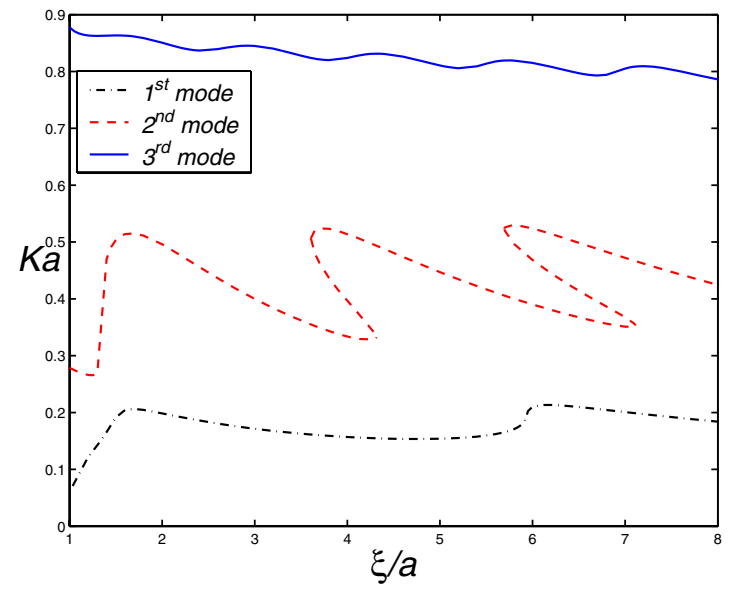

(a)

Fig. 4 a Values of $K a$ for which the real part of the determinant vanishes and $\mathbf{b}$ the absolute values of the determinant of the complex matrix for two cylinders of equal radius $a$ submerged in the lower

that the first and second modes remain unchanged. When the value of $D / a^{4}$ is increased, frequency $K a$ for the third mode decreases. Within the specified range of $\xi / a$, the number of points for which trapped waves exist decreases with an increase in $D / a^{4}$.

Figures 5 and 6 show the variation of these modes with an increase in upper layer depth $d / a$. In both the figures, the submergence depth $f / a$ is considered to be -1.1 and the ice parameters as $D / a^{4}=0.001$ and $\epsilon / a=0.001$. In this case also, there is no variation in the first mode. For the second mode, the values of $\xi / a$ for which trapped waves exist increase corresponding to an increase in the depth of the upper layer. With respect to the third mode, as can be seen from Fig. $6 a$, frequency $K a$ reduces corresponding to an increment in the depth of the upper layer. From Fig. $6 \mathrm{~b}$, it is noticed that corresponding to a decrease in the depth of the upper layer, more points $\xi / a$ occur for which trapped waves exist.

Three different submergence depths are considered in Figs. 7 and 8 as $f / a=-1.05,-1.10,-1.15$. For both the figures, the upper layer depth $d / a$ is taken as 2.0 and both the non-dimensionalized ice parameters are fixed at 0.001 . With the variation of the submergence depth, though the first mode varies but it still does not produce any point on the $\xi / a$-axis for which the absolute value of the determinant vanishes. As a consequence, the first mode does not give rise to any trapped waves within the specified range of $\xi / a$. For the second mode, the values of $\xi / a$, for which trapped waves exist, increase as the submergence depth increases, as can be seen

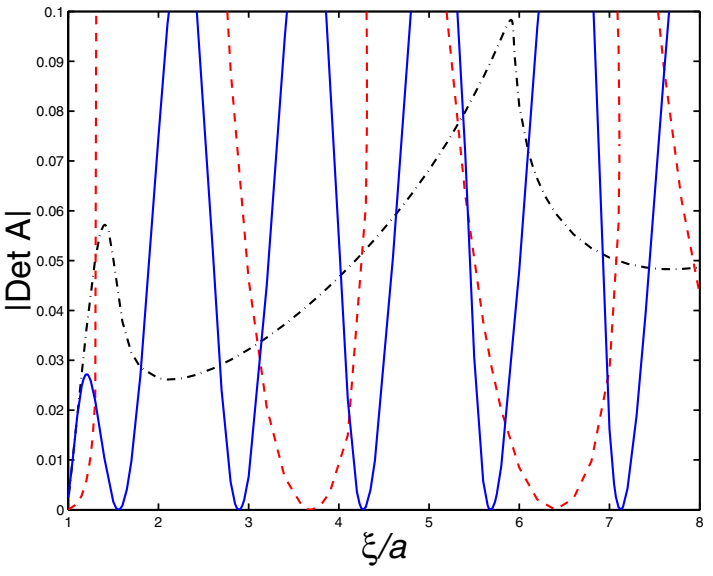

(b)

layer; $\quad d / a=2, f / a=-1.1, \rho=0.5, \alpha_{\text {inc }}=0.34, D / a^{4}=0.01 \quad$ and $\epsilon / a=0.001$

by comparing all the (b) parts of Fig. 7. Figure 8a shows that frequency $\mathrm{Ka}$ for the third mode decreases as $\mathrm{f} / \mathrm{a}$ increases. It can be observed from Fig. $8 \mathrm{~b}$ that the values of $\xi / a$, corresponding to which trapped waves exist, increase when submergence depth increases.

\subsection{Cylinders submerged in the upper layer only}

The pair of identical circular horizontal cylinders is placed in the upper fluid layer $(f>0)$ and hence the work can accomplished completely with potential $\phi^{\prime}$ alone. The symmetric and antisymmetric multipoles, based on cylinder $j, j=1,2$, are given by

$$
\begin{aligned}
\phi_{n j}^{l s}= & K_{n}\left(I r_{j}\right) \cos n \theta_{j}+\int_{0}^{\infty} \cosh n u \cos \left(I x_{j} \sinh u\right) \\
& \times\left[A_{u n}^{(0)}(v) e^{v z_{j}}+B_{u n}^{(0)}(v) e^{-v z_{j}}\right] d u, \\
\phi_{n j}^{l a}= & K_{n}\left(I r_{j}\right) \sin n \theta_{j}+\int_{0}^{\infty} \sinh n u \sin \left(I x_{j} \sinh u\right) \\
& \times\left[A_{u n}^{(1)}(v) e^{v z_{j}}+B_{u n}^{(1)}(v) e^{-v z_{j}}\right] d u,
\end{aligned}
$$

where

$$
\begin{aligned}
& A_{U n}^{(q)}(v)=\frac{F_{+}(v)}{F_{-}(v)} e^{-2 v d}\left((-1)^{n+q} e^{v f}+B_{U n}^{(q)}(v)\right), q=0,1, \\
& B_{U n}^{(q)}(v)=\frac{v-K}{G(v)}\left(F_{-}(v) e^{-v f}+F_{+}(v)(-1)^{n+q} e^{v(f-2 d)}\right) .
\end{aligned}
$$




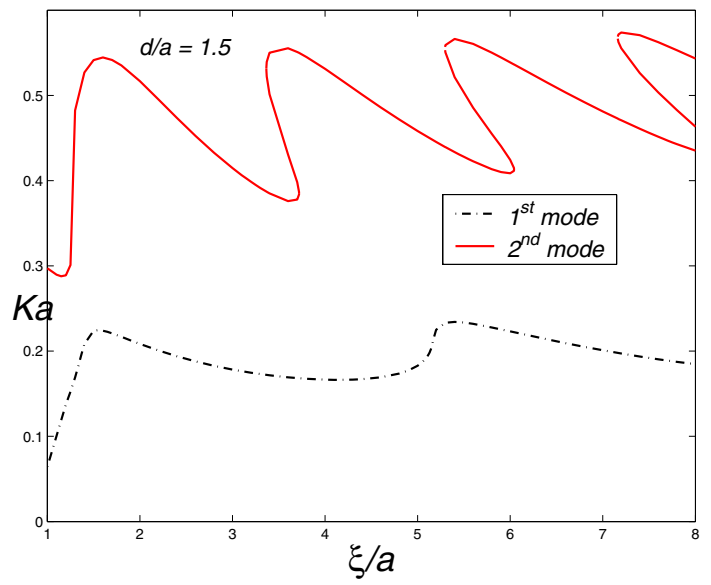

(a)

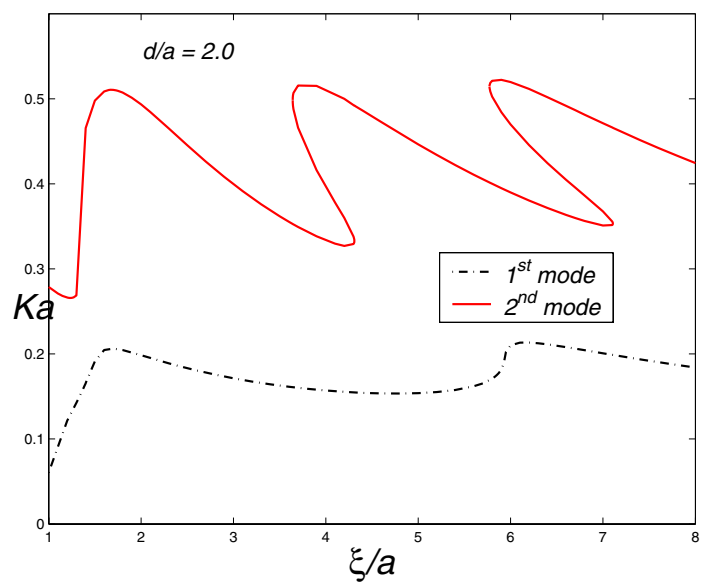

(a)

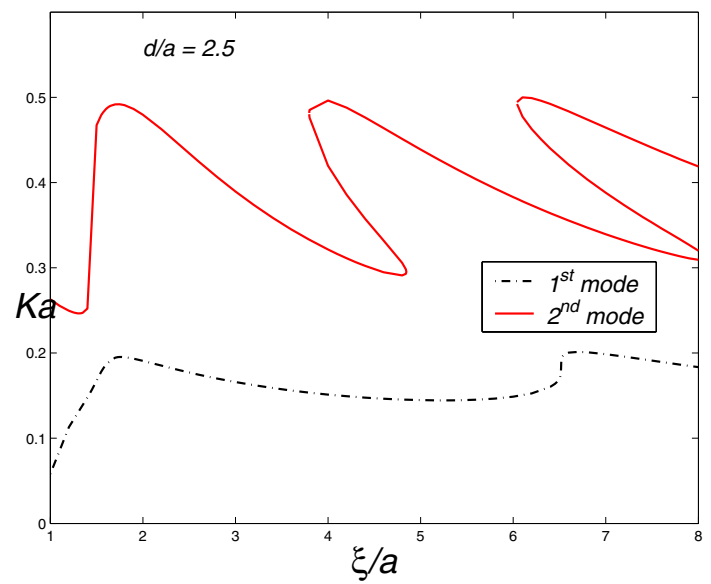

(a)

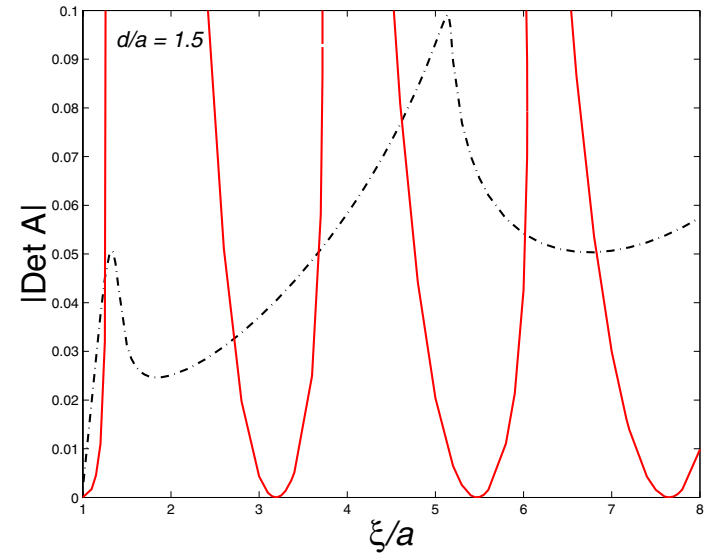

(b)

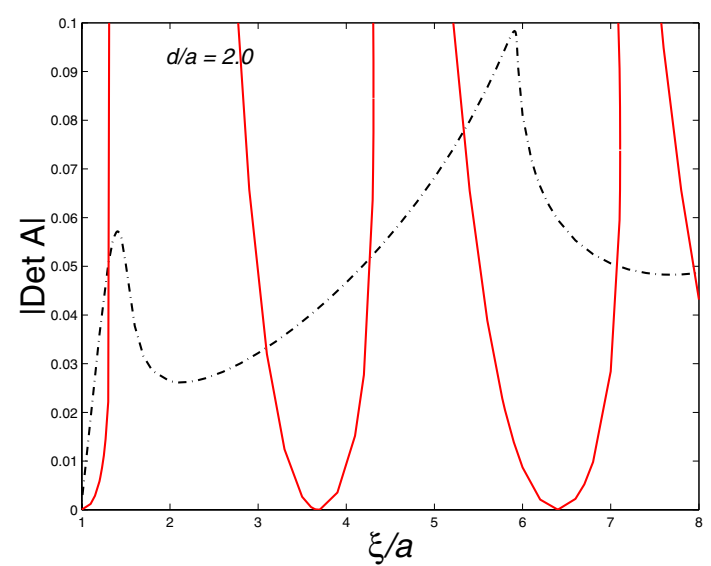

(b)

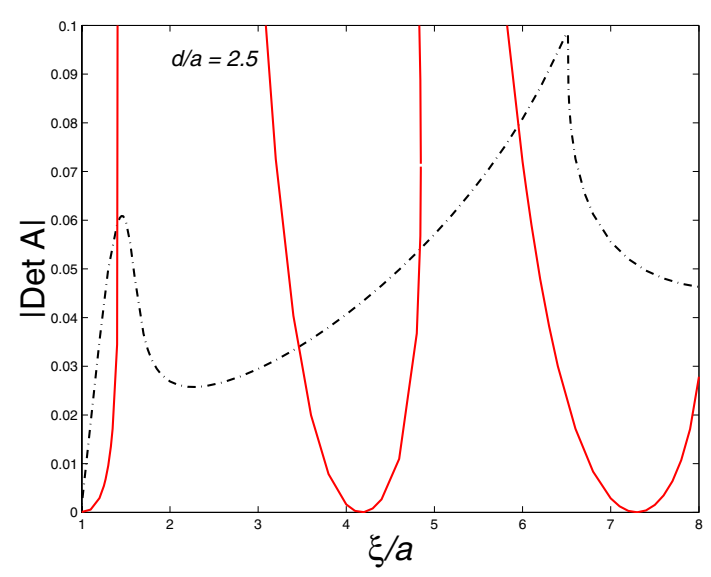

(b) 
4 Fig. 5 a Values of $K a$ for which the real part of the determinant vanishes and $\mathbf{b}$ the absolute values of the determinant of the complex matrix for three different values of upper layer depth $d / a$ for two cylinders of equal radius $a$ submerged in the lower layer; $f / a=-1.1, \rho=0.5, \alpha_{i n c}=0.34, D / a^{4}=0.001$ and $\epsilon / a=0.001$

Proceeding exactly as in the lower layer case, the following infinite system of homogenous linear equations is obtained:

$\alpha_{m}+\frac{I_{m}^{\prime}(l a)}{K_{m}^{\prime}(l a)} \sum_{n=0}^{\infty}\left(P_{n m}^{u} \alpha_{n}+Q_{n m}^{u} \beta_{n}\right)=0$,

$\beta_{m}-\frac{I_{m}^{\prime}(l a)}{K_{m}^{\prime}(l a)} \sum_{n=0}^{\infty}\left(T_{n m}^{u} \beta_{n}+R_{n m}^{u} \alpha_{n}\right)=0, m \geq 0$,

where

$$
\begin{aligned}
P_{n m}^{u}= & \frac{\varepsilon_{m}}{2}\left(K_{n-m}(2 l \xi)+(-1)^{m} K_{n+m}(2 l \xi)\right) \cos (n+m) \frac{\pi}{2} \\
& +\varepsilon_{m} \int_{0}^{\infty} \cosh n u \cosh m u(1+\cos (2 l \xi \sinh u)) \\
& \times\left[(-1)^{m} A_{U n}^{(0)}(v) e^{v f}+B_{U n}^{(0)}(v) e^{-v f}\right] d u, \\
Q_{n m}^{u}= & \frac{\varepsilon_{m}}{2}\left(K_{n-m}(2 l \xi)+(-1)^{m} K_{n+m}(2 l \xi)\right) \sin (n+m) \frac{\pi}{2} \\
& +\varepsilon_{m} \int_{0}^{\infty} \sinh n u \cosh m u \sin (2 l \xi \sinh u) \\
& \times\left[(-1)^{m} A_{U n}^{(1)}(v) e^{v f}+B_{U n}^{(1)}(v) e^{-v f}\right] d u,
\end{aligned}
$$

$$
\begin{aligned}
R_{n m}^{u}= & -\frac{\varepsilon_{m}}{2}\left(K_{n-m}(2 l \xi)+(-1)^{m+1} K_{n+m}(2 l \xi)\right) \sin (n+m) \frac{\pi}{2} \\
& +\varepsilon_{m} \int_{0}^{\infty} \sinh m u \cosh n u \sin (2 l \xi \sinh u) \\
& \times\left[(-1)^{m} A_{u n}^{(0)}(v) e^{v f}-B_{u n}^{(0)}(v) e^{-v f}\right] d u,
\end{aligned}
$$

$$
\begin{aligned}
T_{n m}^{u}= & \frac{\varepsilon_{m}}{2}\left(K_{n-m}(2 l \xi)+(-1)^{m+1} K_{n+m}(2 l \xi)\right) \cos (n+m) \frac{\pi}{2} \\
& +\varepsilon_{m} \int_{0}^{\infty} \sinh n u \sinh m u(\cos (2 / \xi \sinh u-1)) \\
& \times\left[(-1)^{m+1} A_{u n}^{(1)}(v) e^{v f}+B_{u n}^{(1)}(v) e^{-v f}\right] d u .
\end{aligned}
$$

In a similar manner, as in the previous case, by varying the frequency $\mathrm{Ka}$ and fixing the other parameters, we conveniently locate the real zeros of the truncated determinant and then check for the existence of trapped waves by observing the absolute value of the determinant since zeros of the absolute value of the determinant correspond to the trapped modes.

\subsubsection{Numerical results}

With both the cylinders placed in the upper layer only, we investigate the existence of trapped waves with $\xi / a$ varying in the range 1.0 to 6.0 . In this case, only the variation of trapped waves is examined by varying the values of the ice parameters. This consideration is mainly due to the fact that large computational expense will occur while computing the integrals in (4.7)-(4.10) which are more in number as compared to the lower layer case. $\mathrm{Ka}$ is varied up to the value 3.0 to locate the zeros of the real part of the truncated determinant. Here three different

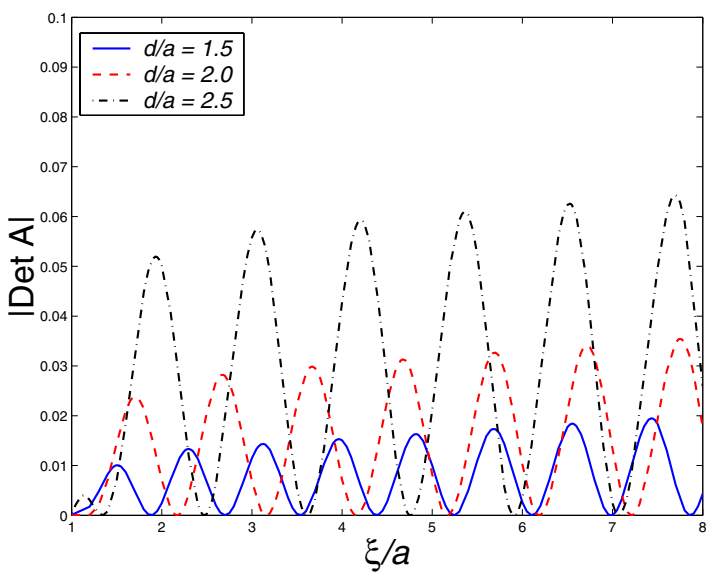

Fig. 6 Variation of third mode for three different values of upper layer depth $d / a$ for two cylinders of equal radius $a$ submerged in the lower layer; $f / a=-1.1, \rho=0.5, \alpha_{\text {inc }}=0.34, D / a^{4}=0.001$ and $\epsilon / a=0.001$ 


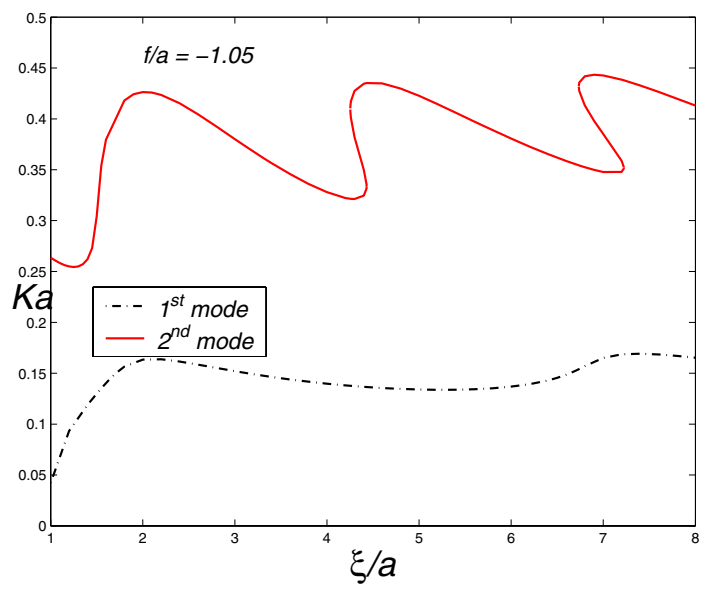

(a)

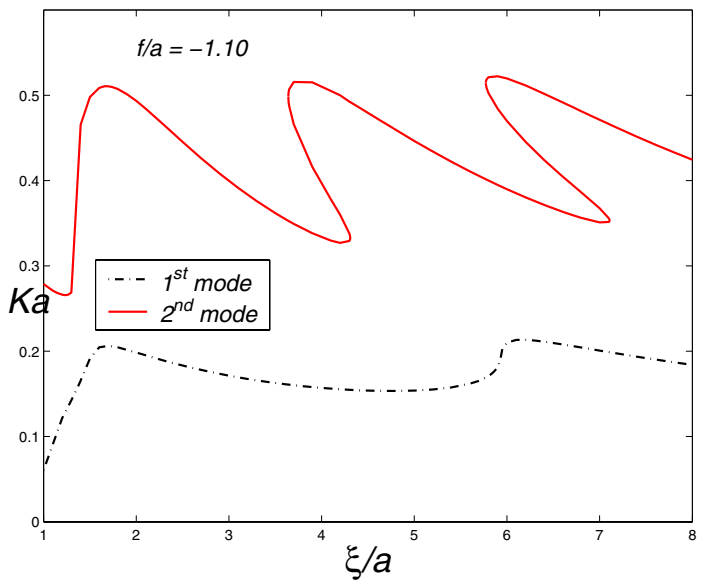

(a)

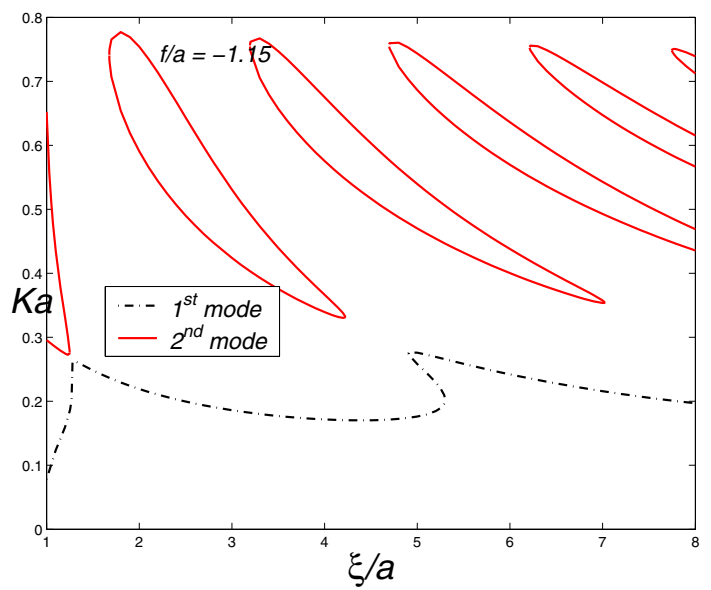

(a)

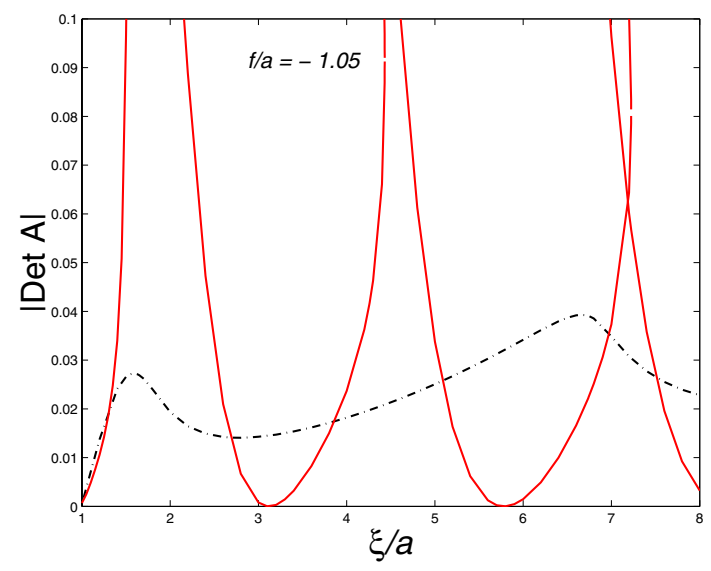

(b)

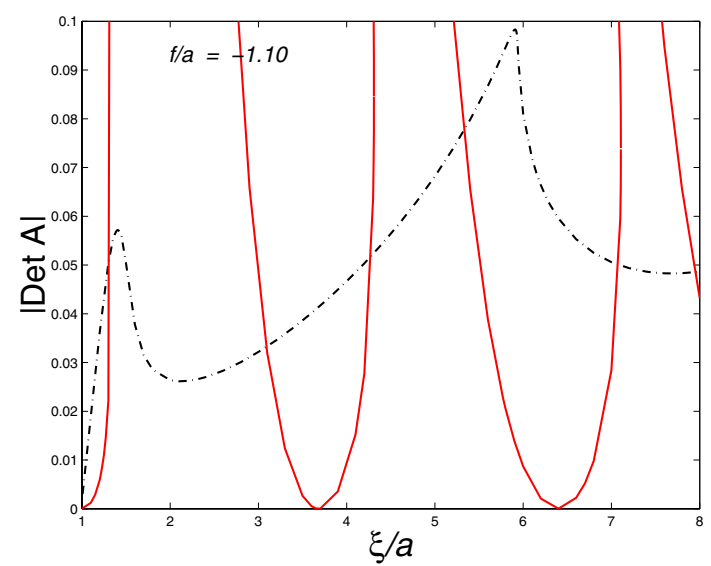

(b)

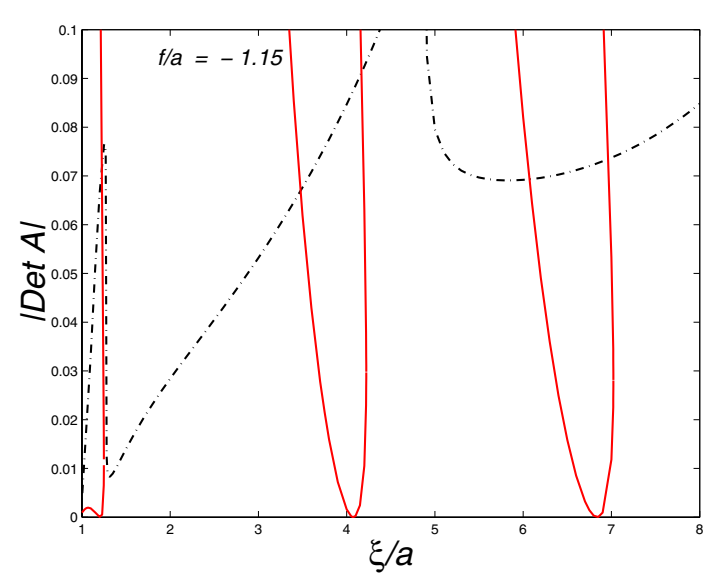

(b) 
4 Fig. 7 a Values of $K a$ for which the real part of the determinant vanishes and $\mathbf{b}$ the absolute values of the determinant of the complex matrix for three different values of submergence depth $\mathrm{f} / \mathrm{a}$ for two cylinders of equal radius $a$ submerged in the lower layer; $d / a=2.0, \rho=0.5, \alpha_{\text {inc }}=0.34, D / a^{4}=0.001$ and $\epsilon / a=0.001$

sets of ice parameters are considered: $D / a^{4}=0, \epsilon / a=0$; $D / a^{4}=0.0001, \epsilon / a=0.0001 ; D / a^{4}=0.001, \epsilon / a=0.001$. The first set will correspond to the result for the upper layer covered by a free surface. In all the figures, $d / a$, the depth of the upper layer, is taken as 2.5 and $f / a$, the submergence depth, as 1.25 .

Figure 9a shows that there exist two modes for which the real part of the determinant vanishes. Corresponding to those values of $K a$, we present the plot of the absolute values of the determinant in Fig. 9b through which it can be observed that for both the modes, there exist values of $\xi / a$ for which trapped waves exist. However, if those values of $\xi / a$ are changed even by a small amount, the embedded trapped waves will cease to exist.

Figure 10 shows that a very small value of the ice parameter $\epsilon / a$ gives rise to one extra mode - the third one, as compared to the case for the problem with a free surface. For this third mode, there exist trapped waves for all values of $\xi / a$ within the range considered. For Fig. 11, the values of the ice parameter set are considered to be 10 times more than those considered for Fig. 10. In this case, the first mode remains the same but the second
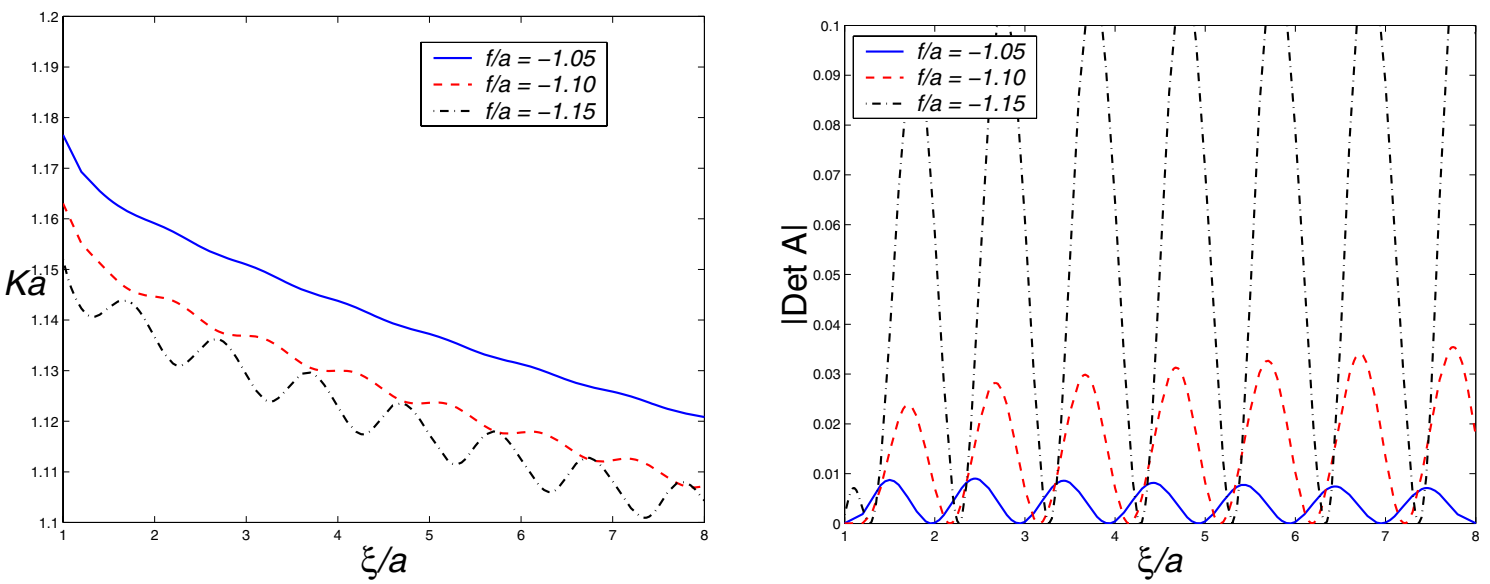

Fig. 8 Variation of third mode for three different values of submergence depth $f / a$ for two cylinders of equal radius $a$ submerged in the lower layer; $d / a=2.0, \rho=0.5, \alpha_{\text {inc }}=0.34, D / a^{4}=0.001$ and $\epsilon / a=0.001$

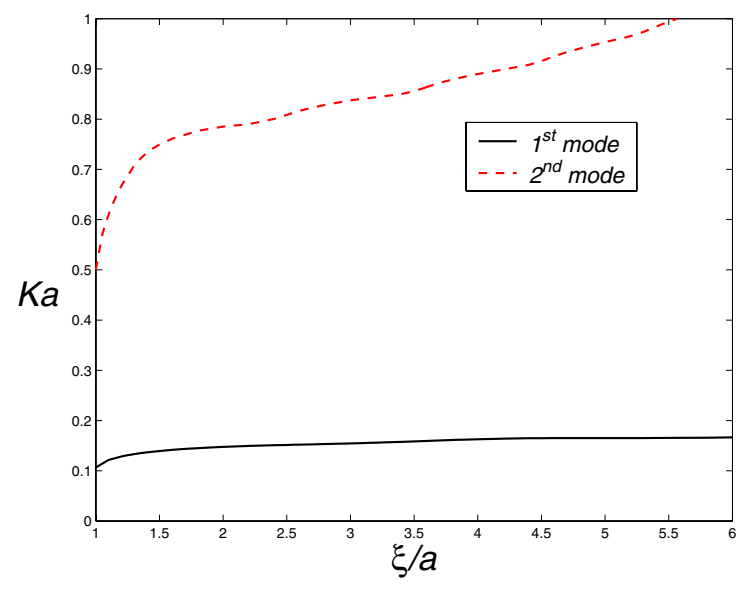

(a)

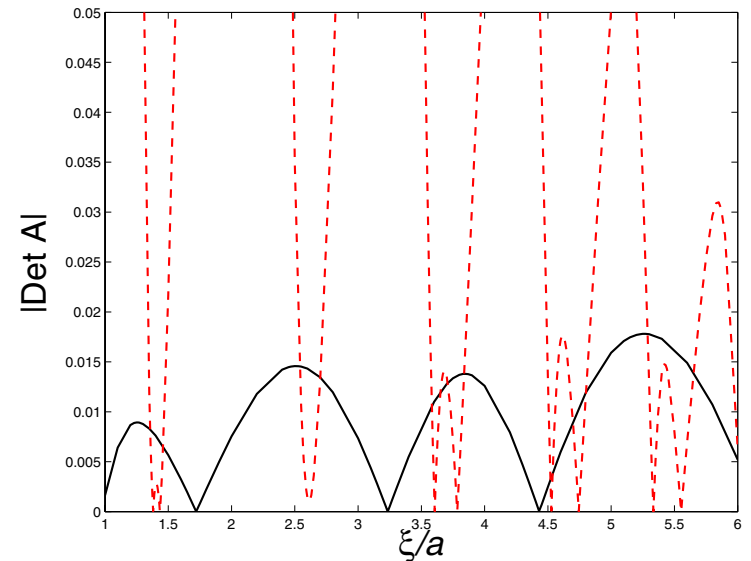

(b)

Fig. 9 a Values of $K a$ for which the real part of the determinant vanishes and $\mathbf{b}$ the absolute values of the determinant of the complex matrix for two cylinders of equal radius $a$ submerged in the upper layer; $d / a=2.50, f / a=1.25, \rho=0.5, \alpha_{i n c}=0.34, D / a^{4}=0$ and $\epsilon / a=0$ 


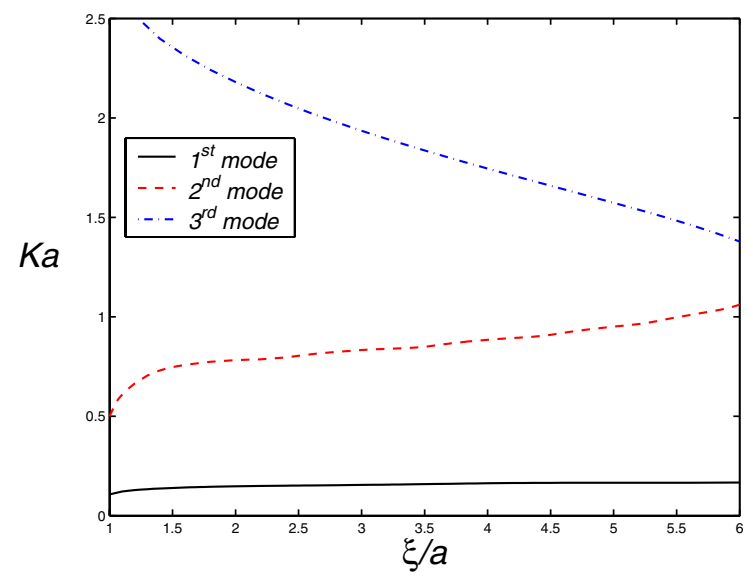

(a)

Fig. 10 a Values of $K a$ for which the real part of the determinant vanishes and $\mathbf{b}$ the absolute values of the determinant of the complex matrix for two cylin-

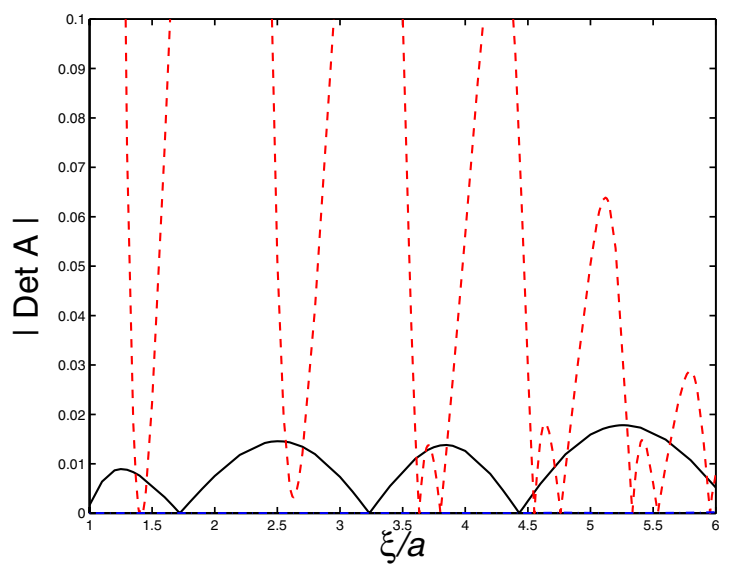

(b)

ders of equal radius $a$ submerged in the upper layer; $d / a=2.50, f / a=1.25, \rho=0.5, \alpha_{i n c}=0.34, D / a^{4}=0.0001 \quad$ and $\epsilon / a=0.0001$

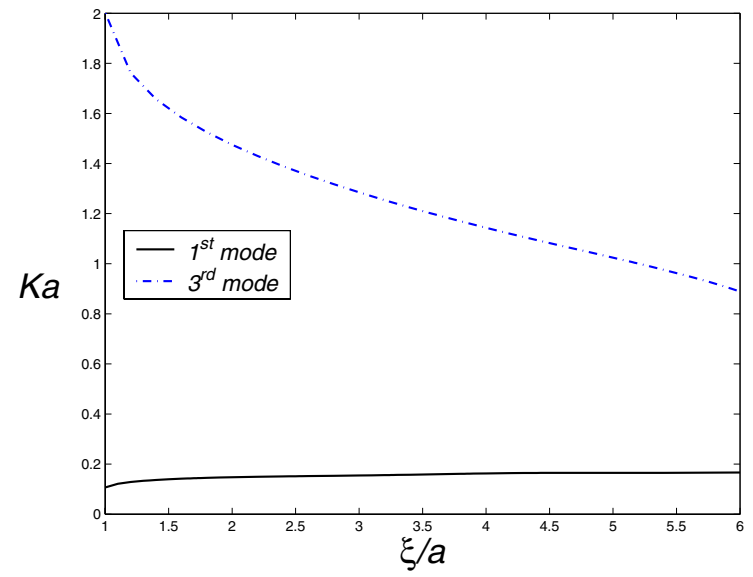

(a)

Fig. 11 a Values of $K a$ for which the real part of the determinant vanishes and $\mathbf{b}$ the absolute values of the determinant of the complex matrix for two cylin-

mode does not exist while the third mode exists but its values get lowered as compared to those in Fig. 10. For the third mode, trapped waves always exist for values of $\xi / a$ approximately up to 3.60 .

\section{Discussion and relevance of present work}

Modeling of large scale atmospheric and oceanic flows in Arctic and Antarctic regions can be accomplished by consideration of flexural gravity waves in a two-layer fluid.

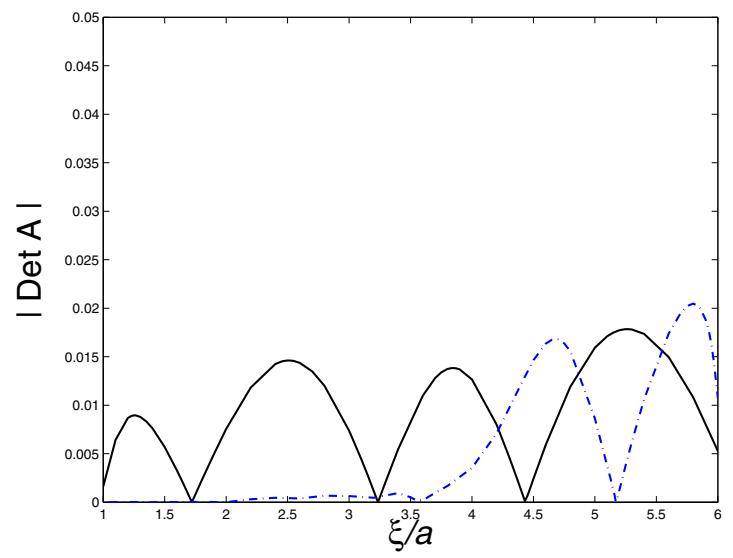

(b)

ders of equal radius $a$ submerged in the upper layer; $d / a=2.50, f / a=1.25, \rho=0.5, \alpha_{i n c}=0.34, D / a^{4}=0.001 \quad$ and $\epsilon / a=0.001$

Existence of embedded trapped waves by a pair of identical circular cylinders submerged in either of the layers of a two-layer fluid is studied in this work. Therefore, it can be claimed that this work sets another example which shows that general uniqueness proof of solution for homogeneous boundary value problem is unobtainable even for a two-layer fluid.

The variations of different parameters locate the region where trapped modes cease to exist and hence unique reflection and transmission coefficient can be determined for that specific set of geometrical configuration of the 
prescribed scattering problem. These trapped wave solutions throw some light on a number of issues related to various aspects of maintenance of the coast or breakwaters and for creation of tranquility zones. The interest in this problem is stimulated by the proposed construction of tube bridges in Norwegian fjords which have a manifestly two-layer structure [Romero Rodriguez and Zhevandrov [30]]. Trapped mode frequencies can be used to estimate the dangerous resonant frequencies that may appear in the scattering problem in the vicinity of the submerged structures. Trapped waves have the potential to lead to sharp gradients in coastal sea level which help in improving the study of ocean currents in North Pacific subtropical gyre and Weddell sea near Antarctica [Hughes et al. [10]].

\section{Conclusion}

The present work is an investigation of trapped water waves due to a pair of horizontal circular cylinders submerged in one of the layers of a two-layer fluid in an ocean, with a finite depth upper layer which is bounded above by a thin ice-cover approximated as a thin elastic plate and an infinite depth lower layer. For such a configuration, for any given frequency, waves propagate at two different wavenumbers - the wave with the smaller wave number is connected to an ice-surface disturbance and the other one to the motion at the interface. Since embedded trapped waves confined only to the area between the cylinders is considered, therefore propagating waves exist only near the interface but not near the ice-cover. When both the cylinders are placed entirely in the lower layer, we present numerically the existence of trapped modes above the cut-off frequency for oblique waves incident on such a geometry. For the considered parameter values, the number of trapped modes enclosed in the continuous spectrum decreases corresponding to an increase in the flexural rigidity of the ice-cover. The trapped mode frequency decreases when either the the upper layer depth or the submergence depth increases. When the cylinders are placed entirely in the upper layer, both cases of the layer covered by a free surface or by a thin ice-cover are considered. Also corresponding to a small change in the separation parameter, embedded trapped modes cease to exist for the free surface and also for a very thin icecover. The present work tries to establish the existence of trapped modes based on numerical evidence only, i.e., the values of those frequencies for which the truncated determinant vanishes are computed. The consideration of two cylinders in either layer in an ice-covered twolayer fluid presents a more challenging problem than the one corresponding to a single cylinder. The present problem of a pair of cylinders can be easily repeated for a system of larger number of cylinders for symmetrical arrangements, say arrays of cylinders. Comparison of present result with an established result of Linton and Cadby [18] shows excellent agreement.

Our investigation is expected to encourage the investigation of various such ocean engineering problems in addition to some related areas of mathematical physics in which higher-order boundary conditions arise in a characteristic manner.

Acknowledgements The authors express their sincere gratitude to the learned Reviewers for their insightful comments which have resulted in a much improved revised version.

\section{Compliance with ethical standards}

Conflict of interest On behalf of both authors, the corresponding author states that there is no conflict of interest.

\section{Appendix: Coordinates shifts}

We are required to transform $K_{n}\left(I r_{j}\right) \cos n \theta_{j}, j=1,2, \ldots, N$, where the coordinates $\left(r_{j}, \theta_{j}\right)$ associated with the $j$-th cylinder are measured from $\left(h_{j}, f\right)$ to the coordinates $\left(r_{p}, \theta_{p}\right)$ associated with the $p$-th cylinder placed at $\left(h_{p}, f\right)$ and vice versa. We use Graf's Addition Theorem for modified Bessel functions ([36]) given by

$$
\begin{aligned}
K_{n}(\beta) \cos n \psi & =\sum_{m=-\infty}^{\infty} K_{n+m}(R) I_{m}(Z) \cos m \phi, \\
K_{n}(\beta) \sin n \psi & =\sum_{m=-\infty}^{\infty} K_{n+m}(R) I_{m}(Z) \sin m \phi,
\end{aligned}
$$

where $R, \beta$ and $Z$ are the sides of the triangle given in Fig. 12. In the present case, the triangle has sides $r_{j}, r_{p}$ and $\left|h_{j}-h_{p}\right|$ and so upon the following substitutions

$\beta=I r_{j} ; \psi=\frac{\pi}{2}-\theta_{j} ; Z=I r_{k} ;$

$\phi=\theta_{k}-\frac{3 \pi}{2} ; R=|| h_{j}-h_{p} \mid$,

and considering the cases $h_{j}>h_{p}, h_{p}>h_{j}$ separately, we have

Fig. 12 Coordinate shift for Graf's addition theorem

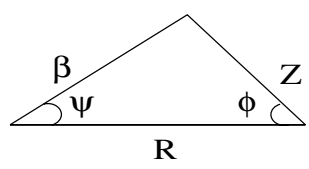

SN Applied Sciences 


$$
\begin{aligned}
K_{n}\left(I r_{j}\right) \cos n \theta_{j} & =\sum_{m=0}^{\infty}\left(C_{n m}^{j p} \cos m \theta_{p}+D_{n m}^{j p} \sin m \theta_{p}\right) I_{m}\left(I r_{p}\right), \\
K_{n}\left(I r_{j}\right) \sin n \theta_{j} & =\sum_{m=0}^{\infty}\left(A_{n m}^{j p} \cos m \theta_{p}+B_{n m}^{j p} \sin m \theta_{p}\right) I_{m}\left(I r_{p}\right),
\end{aligned}
$$

where

$$
\begin{aligned}
A_{n m}^{j p} & =\frac{\varepsilon_{m}}{2}\left((-1)^{m} K_{n+m}\left(I\left|h_{j}-h_{p}\right|\right)+K_{n-m}\left(I\left|h_{j}-h_{p}\right|\right)\right) \sin (n+m) \frac{\pi}{2}, \\
B_{n m}^{j p} & =\frac{\varepsilon_{m}}{2}\left((-1)^{m+1} K_{n+m}\left(I\left|h_{j}-h_{p}\right|\right)+K_{n-m}\left(I\left|h_{j}-h_{p}\right|\right)\right) \cos (n+m) \frac{\pi}{2}, \\
C_{n m}^{j p} & =\frac{\varepsilon_{m}}{2}\left((-1)^{m} K_{n+m}\left(I\left|h_{j}-h_{p}\right|\right)+K_{n-m}\left(I\left|h_{j}-h_{p}\right|\right)\right) \cos (n+m) \frac{\pi}{2}, \\
D_{n m}^{j p} & =-\frac{\varepsilon_{m}}{2}\left((-1)^{m+1} K_{n+m}\left(I\left|h_{j}-h_{p}\right|\right)+K_{n-m}\left(I\left|h_{j}-h_{p}\right|\right)\right) \sin (n+m) \frac{\pi}{2} .
\end{aligned}
$$

Using the relations $x_{j}=h_{p}-h_{j}+x_{k}$ and $z_{j}=z_{p}$ in the integral in (3.3), we obtain

$$
\begin{aligned}
& (-1)^{n} \int_{0}^{\infty} \cosh n u \cos \left(\mid x_{j} \sinh u\right) e^{v z_{j}} C_{L}(v) d u \\
& =(-1)^{n} \int_{0}^{\infty} \cosh n u \cos \left(I\left|h_{p}-h_{j}\right| \sinh u\right) \cos \left(\mid x_{p} \sinh u\right) e^{v z_{p}} C_{L}(v) d u \\
& +(-1)^{n+1} \int_{0}^{\infty} \cosh n u \sin \left(I\left|h_{p}-h_{j}\right| \sinh u\right) \times \sin \left(\mid x_{p} \sinh u\right) e^{v z_{p}} C_{L}(v) d u
\end{aligned}
$$

The well known generating function of modified Bessel functions is utilized [36]:

$\exp \left[\frac{1}{2} X\left(T+T^{-1}\right)\right]=\sum_{m=0}^{\infty} \frac{1}{2} \varepsilon_{m}\left(T^{m}+T^{-m}\right) I_{m}(X)$,

where $\varepsilon_{0}=1, \varepsilon_{m}=2, m \geq 1$. Substituting $X=-l r_{p}$, $T=\exp \left[i\left(\theta_{p}+i u\right)\right]$ in it and equating real and imaginary parts, the following results are obtained:

$$
\begin{aligned}
& e^{v z_{p}} \cos \left(I x_{p} \sinh u\right)=e^{v f} \sum_{m=0}^{\infty}(-1)^{m} \varepsilon_{m} \cosh m u I_{m}\left(I r_{p}\right) \cos m \theta_{p^{\prime}} \\
& e^{v z_{p}} \sin \left(I x_{p} \sinh u\right)=e^{v f} \sum_{m=0}^{\infty}(-1)^{m+1} \varepsilon_{m} \sinh m u I_{m}\left(I r_{p}\right) \sin m \theta_{p} .
\end{aligned}
$$

Using the above relations in (A.3), we obtain

$$
\begin{aligned}
(-1)^{n} \int_{0}^{\infty} \cosh n u \cos \left(I x_{j} \sinh u\right) e^{v z_{j}} C_{L}(v) d u \\
=\sum_{m=0}^{\infty}\left[\alpha_{n m}^{j p} \cos m \theta_{p}+\beta_{n m}^{j p} \sin m \theta_{p}\right] I_{m}\left(I r_{p}\right)
\end{aligned}
$$

with

$$
\begin{aligned}
& \alpha_{n m}^{j p}=(-1)^{m+n} \varepsilon_{m} \int_{0}^{\infty} \cosh n u \cosh m u \cos \left(|| h_{p}-h_{j} \mid \sinh u\right) e^{v f} C_{L}(v) d u, \\
& \beta_{n m}^{j p}=(-1)^{m+n} \varepsilon_{m} \int_{0}^{\infty} \cosh n u \sinh m u \sin \left(I\left|h_{p}-h_{j}\right| \sinh u\right) e^{v f} C_{L}(v) d u .
\end{aligned}
$$

Similarly, for the integral in (3.5), we get

$$
\begin{gathered}
(-1)^{n+1} \int_{0}^{\infty} \sinh n u \sin \left(I x_{j} \sinh u\right) e^{v z_{j}} C_{L}(v) d u \\
=\sum_{m=0}^{\infty}\left[a_{n m}^{j p} \cos m \theta_{p}+b_{n m}^{j p} \sin m \theta_{p}\right] I_{m}\left(I r_{p}\right),
\end{gathered}
$$

with

$$
\begin{aligned}
& a_{n m}^{j p}=(-1)^{m+n+1} \varepsilon_{m} \int_{0}^{\infty} \sinh n u \cosh m u \sin \left(I\left|h_{p}-h_{j}\right| \sinh u\right) e^{v f} C_{L}(v) d u, \\
& b_{n m}^{j p}=(-1)^{m+n} \varepsilon_{m} \int_{0}^{\infty} \sinh n u \sinh m u \cos \left(I\left|h_{p}-h_{j}\right| \sinh u\right) e^{v f} C_{L}(v) d u .
\end{aligned}
$$

\section{References}

1. Abramowitz M, Stegun IA (1964) Handbook of mathematical functions with formulas, graphs, and mathematical tables. Dover, New York

2. Chung H, Fox $C$ (2002) Calculation of wave-ice interaction using Weiner-Hopf technique. N Z J Math 31(1):1-18

3. Davies EB, Parnovski L (1998) Trapped modes in acoustic waveguides. Q J Mech Appl Math 51(3):477-492

4. Harter R, Abrahams ID, Simon MJ (2007) The effect of surface tension on trapped modes in water-wave problem. Proc $\mathrm{R}$ Soc A 463:3131-3149

5. Harter R, Simon MJ, Abrahams ID (2008) The effect of surface tension on localized free-surface oscillations about surfacepiercing bodies. Proc R Soc A 464:3039-3054

6. Das D, Mandal BN (2007) Wave scattering by a horizontal circular cylinder in a two-layer fluid with an ice-cover. Int J Eng Sci 45:842-872

7. Evans DV, Levitin M, Vassiliev D (1994) Existence theorem for trapped modes. J Fluid Mech 261:21-31

8. Evans DV, Porter R (2003) Wave scattering by narrow cracks in ice sheets floating on water of finite depth. J Fluid Mech 484:143-165

9. Fox C, Squire VA (1994) On the oblique reflection and transmission of ocean waves at shore fast sea ice. Philos Trans R Soc Lond A 347:185-218

10. Hughes CW, Fukumori I, Griffies SM, Huthnance JM, Minobe $S$, Spence P, Thompson KR, Wise A (2019) Sea level and the role of caostal trapped waves in mediating the influence of the open ocean on the coast. Sur Geophys 40:1467-1492

11. Kassem SE (1982) Multipole expansions for two superposed fluids, each of finite depth. Math Proc Camb Philos Soc 91:323-329

12. Kuznetsov N (1993) Trapping modes of internal waves in a channel spanned by a submerged cylinder. J Fluid Mech 254:113-126

13. Kuznetsov N, Porter R, Evans DV, Simon MJ (1998) Uniqueness and trapped modes for surface-piercing cylinders in oblique waves. J Fluid Mech 365:351-368

14. Kuznetsov N, Maz'ya V, Vainberg B (2008) Linear water waves: a mathematical approach. Cambridge University Press, United Kingdom

15. Linton CM, Evans DV (1992) Integral equations for a class of problems concerning obstacles in waveguides. J Fluid Mech 245:349-365

16. Linton CM, Evans DV (1992) The radiation and scattering of surface waves by a vertical circular cylinder in a channel. Philos Trans R Soc Lond A 338:325-357

17. Linton CM, Cadby JR (2002) Scattering of oblique waves in a two-layer fluid. J Fluid Mech 461:343-364 
18. Linton CM, Cadby JR (2003) Trapped modes in a two-layer fluid. J Fluid Mech 481:215-234

19. Linton CM, Mclver $P$ (2007) Embedded trapped modes in water waves and acoustics. Wave Motion 45:16-29

20. Lu DQ, Sun CZ (2013) Transient flexural- and capillary-gravity waves due to disturbances in a two-layer density-stratified fluid. J HYdro 25(3):339-347

21. Mclver P, Evans DV (1985) The trapping of surface waves above a submerged horizontal cylinder. J Fluid Mech 151:242-255

22. Mclver M (1996) An example of non-uniqueness in the two-diemensional linear water wave problem. J Fluid Mech 315:257-266

23. Motygin OV (1999) Trapped modes of oscillation of a liquid for surface-piercing bodies in oblique waves. J Appl Math Mech 63:257-264

24. Mohapatra S, Bora SN (2009) Propagation of oblique waves over small bottom undulation in an ice-covered two-layer fluid. Geophys Astro Fluid 103(5):347-374

25. Mohapatra S, Bora SN (2012) Exciting forces due to interaction of water waves with a submerged sphere in an ice-covered twolayer fluid of finite depth. Appl Ocean Res 34:187-197

26. Nazarov SA, Videman JH (2009) A sufficient condition for the existence of trapped modes for oblique waves in a two-layer fluid. Proc R Soc Lond A 465:3799-3816

27. Nazarov SA, Taskinen J, Videman JH (2013) Asymptotic behavior of trapped modes in two-layer fluids. Wave Motion 50:111-126

28. Porter R, Evans DV (1998) The trapping of surface waves by multiple submerged horizontal cylinders. J Eng Math 34:417-433
29. Porter R (2002) Trapping of water waves by pairs of submerged cylinders. Proc R Soc Lond A 458:607-624

30. Romero Rodriguez MI, Zhevandrov P (2019) Water waves trapped by thin submerged cylinders in a two-layer fluid: discrete eigenvalue. Math Meth Appl Sci 42:4999-5007

31. Simon MJ, Ursell F (1984) Uniqueness in linearized two-dimensional water-wave problems. J Fluid Mech 148:137-154

32. Bhattacharjee J, Sahoo T (2008) Flexural gravity wave problems in two-layer fluids. Wave Motion 45:133-153

33. Saha S, Bora SN (2013) Trapped modes in a two-layer fluid of finite depth bounded above by a rigid lid. Wave Motion 50:1050-1060

34. Saha S, Bora SN (2014) Flexural gravity waves trapped in a twolayer fluid of finite depth. Appl Ocean Res 44:1-12

35. Tabssum S, Kaligatla RB, Sahoo T (2015) Gravity wave interaction with a porous breakwater in a two-layer ocean of varying depth. Ocean Eng 196:106816

36. Watson GN (2008) A treatise on the theory of Bessel functions. Cambridge University Press, United Kingdom

37. Xu F, Lu DQ (2010) Wave scattering by a thin elastic plate floating on a two-layer fluid. Int J Eng Sci 48(9):809-819

Publisher's Note Springer Nature remains neutral with regard to jurisdictional claims in published maps and institutional affiliations. 